\title{
TV/Series
}

11 | 2017

Philosopher avec Battlestar Galactica

\section{Destin, Providence et temps cyclique : Quelle liberté pour les personnages de Battlestar Galactica ?}

Isabelle Périer

\section{OpenEdition}

Journals

Édition électronique

URL : http://journals.openedition.org/tvseries/2096

DOI : 10.4000/tvseries.2096

ISSN : 2266-0909

Éditeur

GRIC - Groupe de recherche Identités et Cultures

Référence électronique

Isabelle Périer, «Destin, Providence et temps cyclique : Quelle liberté pour les personnages de Battlestar Galactica? », TV/Series [En ligne], 11 | 2017, mis en ligne le 09 juillet 2017, consulté le 01 mai 2019. URL : http://journals.openedition.org/tvseries/2096 ; DOI : 10.4000/tvseries.2096

Ce document a été généré automatiquement le 1 mai 2019.

\section{(c) (1)}

TV/Series est mis à disposition selon les termes de la licence Creative Commons Attribution - Pas d'Utilisation Commerciale - Pas de Modification 4.0 International. 


\title{
Destin, Providence et temps cyclique : Quelle liberté pour les personnages de Battlestar Galactica?
}

\author{
Isabelle Périer
}

1 La série Battlestar Galactica, dans sa réécriture par Ronald D. Moore (Syfy, 2003-2009), est une série de science-fiction combinant technologie avancée - suffisamment avancée pour que les hommes aient pu donner naissance à des intelligences artificielles douées de conscience - et religion. Les questions du sacré et de la providence semblent en effet centrales dans une série où la plupart des personnages s'interrogent sur leur destin. Ainsi, la première saison est, entre autres, le récit de la conversion de Baltar, le scientifique rationaliste, de sa prise de conscience de l'existence de Dieu ${ }^{1}$ et d'une providence qui guide la destinée de chacun; c'est en même temps celle de la révélation qui s'impose à la présidente Roslin du caractère cyclique du temps, de la répétition de l'histoire et du rôle qu'elle-même doit y jouer. Cette étude se propose donc d'étudier la conception du temps, du destin et de la liberté à l'œuvre dans la série en la confrontant à la cosmologie cyclique et providentielle des stoïciens. Dans la mesure où l'intertextualité à l'œuvre dans la série est une manière de représenter les déterminismes internes et externes pesant sur les personnages en créant, par un effet de « déjà-vu », l'impression d'un éternel retour, nous nous appuierons aussi sur une analyse mythocritique pour saisir les enjeux philosophiques et cosmologiques de ce récit, qui propose finalement, dans son déplacement d'une conception circulaire vers une conception « spiralaire » du temps, une conclusion en forme d'ouverture sur d'autres futurs possibles.

3 La série Battlestar Galactica relevant du genre de la science-fiction et présentant une société apparemment futuriste où la technologie est avancée, les spectateurs sont tentés de lui appliquer la conception du temps dominante et courante qui est la nôtre lorsque nous abordons des récits, à savoir celle d'un temps linéaire ${ }^{2}$. De plus, les affirmations de Baltar face à l'apparition de Numéro Six, répétant qu'il est un homme rationnel et un 
scientifique (S01E01), tendent à renforcer ce présupposé. Pourtant, cette conception linéaire du temps est rapidement mise en question, et ce, à plusieurs reprises. Cette remise en cause culmine avec les révélations de Leoben à Kara (S01E08). Leoben, par la formule répétée tout au long de la série ( All of this has happened before and will happen again »), sous-entend que le temps est cyclique et que les événements se répètent à l'identique à chaque cycle ${ }^{3}$. Il se montre d'autant plus convaincant qu'il délivre également une prophétie à Kara, qui s'avérera dans la suite de la série. En outre, les cylons revendiquent leur foi monothéiste en un Dieu unique, s'opposant ainsi aux humains qui sont polythéistes et révèrent les douze dieux de Kobol. Certains cylons, comme Numéro Six et son apparition qui hante Baltar, semblent même extrêmement pieux et ne cessent de proclamer l'intervention de Dieu dans le monde. Ainsi, Numéro Six affirme régulièrement à Baltar que les événements qu'il doit affronter sont le plan de Dieu ${ }^{4}$. Cette référence à un Dieu invisible, transcendant et providentiel se double d'un leitmotiv, celui du destin, de plus en plus important à mesure que passent les épisodes. Nombre de personnages, comme Baltar, la présidente Laura Roslin ou Kara Thrace, affirment que leur destin participe d'un plan divin, qu'il s'agisse du Dieu unique des cylons pour Baltar, ou des douze dieux de Kobol pour la présidente.

4 Or, ces affirmations ne sont pas de vaines paroles - y compris si on les entend comme étant, au départ, pur espoir ou parole qui se veut performative, davantage que prophétie au sens réel du terme, dans le cas de la présidente notamment. De nombreux d'épisodes tendent à développer l'idée que l'univers de Battlestar Galactica est dirigé par une forme de providence divine qui agence les événements et leur donne un sens, parfois déchiffrable et perceptible par les humains, obscur la plupart du temps 5 . Seule la fin de la série donne sens à tous ces événements improbables qui semblent être le fait du hasard et qui se révèlent néanmoins, en dernier lieu, nécessaires pour que le destin conjoint de l'humanité et des cylons s'accomplisse ${ }^{6}$. Ainsi le moindre détail peut se révéler un indice ou un fragment de la gigantesque énigme posée aux personnages (comme aux spectateurs). Par exemple, les peintures de Kara, aperçues dès la deuxième saison sur Caprica, lorsqu'elle revient dans son appartement en compagnie d'Agathon, sont déjà, à son insu, la représentation de la nova qui va les conduire à la nébuleuse, représentation que retrouvera Tyrol dans le temple des Cinq dieux (S03E12). Cette conjonction d'une conception circulaire du temps, de la présence d'un Dieu unique et transcendant, de la providence et du destin semble très proche de la conception stoïcienne du temps et du cosmos, même si iconographiquement, le dessin de Kara se rapproche aussi d'un mandala.

En effet, voici comment Némésius présente la conception du cosmos des stoïciens :

Les Stoïciens disent que, lorsque les planètes retournent au même signe [zodiacal], en longueur et largeur, que celui auxquelles étaient au début, quand le monde commença à se former, elles produisent, en des périodes de temps déterminées, l'embrasement et la destruction de toutes choses existantes. Puis de nouveau, le monde retourne au même état; et lorsque les astres se meuvent de nouveau de la même façon, chacun des événements qui se sont produits dans la période antérieure se réalisera sans aucun écart : il y aura une fois encore Socrate, et Platon, et chacun des hommes, avec les mêmes amis et concitoyens; ils subiront les mêmes choses, il leur arrivera les mêmes choses, ils s'adonneront aux mêmes choses ; toutes les cités, tous les villages, tous les champs reviendront pareillement. Le retour de toutes choses ne se produit pas une seule fois, mais plusieurs fois; ou plutôt, les mêmes choses reviennent à l'infini et sans jamais s'arrêter. Les dieux, qui ne sont pas sujets à la destruction, ayant suivi de près l'unique période 
présente, connaissent à partir d'elle tout ce qui se passera dans les périodes suivantes, car il n'y aura rien d'étranger à ce qui s'est passé antérieurement : toutes choses se répéteront pareillement, sans aucun écart jusqu'aux moindres détails ${ }^{7}$.

6 Cette conception du cosmos et d'un temps circulaire qui se répète à l'identique à chaque cycle cosmique pose, depuis l'Antiquité, la question de la liberté, du sens de l'action et de la responsabilité morale. En effet, si tout est destiné à s'accomplir à chaque fois à l'identique et que le destin de chacun est déterminé depuis la naissance - ou plutôt la renaissance - du cosmos, à quoi bon agir ? C'est ce que la philosophie stoïcienne a nommé l'argument paresseux, argument auquel répond Cicéron dans son traité Du destin :

Nous ne nous laisserons pas non plus entraver par ce qu'on appelle l'Argument Paresseux (l'argos logos, comme le désignent les philosophes) ; si nous l'écoutions, nous n'agirions absolument pas dans la vie. On le formule ainsi : "Si c'est ton destin de guérir de cette maladie, tu en guériras, que tu fasses venir le médecin ou non; de même, si c'est ton destin de ne pas guérir de cette maladie, tu n'en guériras pas, que tu fasses venir le médecin ou non. Or l'un ou l'autre est ton destin. Donc cela n'a aucun intérêt de faire venir le médecin » [...]. Chrysippe critique cet argument. Il y a dans le monde, dit-il, des faits simples et d'autres qui sont couplés. "Socrate mourra tel jour » est simple ; le jour de sa mort est fixé, quoi qu'il fasse ou ne fasse pas. Mais si le destin prévoit « ÆEdipe naîtra de Laïos », on ne pourra pas dire « que Laïos ait été ou non avec une femme »; car il s'agit d'un fait couplé et " confatal ». Il use de ce terme parce que ce que le destin prévoit, c'est à la fois que Laïos couchera avec sa femme et qu'il engendrera d'elle đEdipe. C'est comme si l'on avait dit «Milon combattra aux Jeux Olympiques» : si quelqu'un répliquait : «donc, qu'il ait un adversaire ou non, il combattra », il se tromperait. En effet, « il combattra » est un fait couplé, parce que sans adversaire il n'y a pas de combat. Tous les sophismes de ce genre se réfutent donc de la même façon. "Tu guériras, que tu fasses venir le médecin ou non » est sophistique, car il est prévu par le destin que tu fasses venir le médecin, tout autant qu'il l'est que tu guérisses. C'est cela, comme je l'ai dit, que Chrysippe appelle les choses confatales ${ }^{8}$.

7 De fait, les personnages de Battlestar ne sont jamais tentés par l'argument paresseux : la perspective de l'échec les hante. Ce besoin d'agir est même parfois perçu comme pathologique et irrationnel, comme dans le cas de Kara, revenue d'entre les morts, qui veut absolument revenir à la nébuleuse pour retrouver le chemin de la Terre, et va jusqu'à menacer la Présidente. Kara manifeste une souffrance à la fois psychologique, spirituelle et physique à chaque saut spatial qui éloigne le Battlestar de la nébuleuse, sentant clairement qu'elle s'écarte de son destin. De même, lorsque Baltar est tenté de baisser les bras et de cesser d'agir, l'apparition de Numéro Six lui répète qu'il est entre les mains de Dieu et qu'il doit l'aider à accomplir son plan, donnant ainsi un sens à ses actes en tant qu'ils participent de son destin (elle souligne au passage que s'il fait partie, sans le savoir, des « Cinq Derniers » cylons, son sentiment de panique fait simplement partie de sa programmation et de sa "couverture » qui masque son identité, y compris à luimême).

8 La question du déterminisme et de la part de liberté laissée aux hommes dépend en partie du courant du stoïcisme auquel on se réfère : 
Les anciens philosophes ont pris deux partis. Il y a eu ceux qui pensaient que tout arrive par le destin, d'une manière telle que ce destin apporte la force de la nécessité. [...]

Les partisans de l'autre position croyaient qu'il existe des mouvements volontaires de notre esprit, libres de tout destin. [...]

Leur raisonnement est le suivant: «Si tout arrive par le destin, tout arrive en vertu d'une cause antécédente. Et si c'est le cas pour l'impulsion, c'est aussi le cas pour ce qui suit de l'impulsion; donc c'est le cas pour les actes d'assentiment. Mais si la cause de l'impulsion ne réside pas en nous, l'impulsion, elle non plus, n'est pas en notre pouvoir. Mais s'il en est ainsi, les résultats eux-mêmes de l'impulsion ne sont pas en notre pouvoir. Donc, ni les actes d'assentiment ni les actions ne sont en notre pouvoir. Il en résulte que ni les louanges, ni les blâmes, ni les honneurs, ni les punitions ne sont justes. » Puisque ce raisonnement est vicieux, ils considèrent comme une inférence probable que tout n'arrive pas par le destin?.

9 Ainsi, la conception d'un temps cyclique éternellement semblable et entièrement déterminé pose la question éthique de la responsabilité morale. Comme l'idée d'une irresponsabilité humaine, notamment face au mal, paraît irrecevable et incompatible avec l'ordre du monde réglé par la divinité, les stoïciens ont souvent tenté d'assouplir cette idée de déterminisme afin d'ouvrir la possibilité d'une responsabilité morale.

Toutefois, le cas des personnages de Battlestar Galactica est particulier, en ce qu'ils s'inscrivent dans un cosmos et une temporalité qui ne sont pas uniquement stoïciens, mais, tout comme les personnages clés de la série, «hybrides ». En effet, si le temps de l'univers de Battlestar est bien cyclique et induit un éternel retour, sa cosmologie est en grande partie teintée de christianisme.

11 Tout d'abord, si la référence aux douze dieux de Kobol, qui portent les noms du panthéon olympien ou du moins de la tradition grecque, ancre cette cosmologie dans un contexte païen, l'insistance des cylons, et notamment de Numéro Six, puis de Baltar, à affirmer que seul existe «le Dieu unique» («the one true God»), infléchit cette cosmogonie vers le monothéisme. Certes, les stoïciens parlaient indifféremment d'un Dieu, des dieux ou même de Zeus ${ }^{10}$. Toutefois, cet infléchissement vers le monothéisme se double d'une insistance troublante de Numéro Six : Baltar doit non seulement croire en son destin et agir de manière à l'accomplir, mais il ne pourra être sauvé que s'il fait acte de foi. C'est précisément ce que montre l'épisode S01E07: après s'être moqué de la croyance de Numéro Six, Baltar est abandonné par sa vision et accusé de trahison par une certaine Shelley Godfrey, qui n'est autre que l'un des clones de Numéro Six. Baltar est ensuite providentiellement acquitté après une longue prière dans laquelle il reconnaît la providence divine et se soumet à la volonté de Dieu. Cet acquittement est d'autant plus étrange et providentiel que Shelley Godfrey disparait mystérieusement une fois Baltar acquitté ${ }^{11}$. L'efficience de la foi est non seulement affirmée par Numéro Six, mais elle est à de nombreuses reprises "démontrée " à Baltar le sceptique. Par exemple, à l'épisode S01E10, le scientifique doit montrer l'emplacement d'une raffinerie de Thylium alors qu'il ignore absolument à quoi elle peut ressembler. Numéro Six lui suggère, lorsqu'elle lui apparaît en vision, de s'en remettre à Dieu. Il désigne donc un point au hasard, devenant, comme il le verbalise lui-même à la fin de l'épisode, l'« instrument » de la volonté divine. Cet épisode montre que les notions de destin et de providence, contrairement à la cosmologie stoïcienne, sont liées à la notion de foi : c'est sa foi qui transforme un geste 
fait au hasard en information providentielle destinée à sauver toute la flotte. Ce lien entre destin, providence et foi donne une coloration chrétienne affirmée à la série.

Sur le plan du temps, le cylon Leoben fournit une information capitale aux spectateurs comme aux humains intradiégétiques lors de sa révélation cosmologique (S01E08) : si les événements se répètent, ils ne se répètent pas exactement à l'identique. Il révèle en effet à Kara que, si les événements se répètent, les rôles des uns et des autres peuvent changer. Cette importance de la variation sur le même est également suggérée lors de l'anamnèse du colonel Tigh sur Terre (S04E11), alors qu'il voit Ellen mourir dans ses bras, d'abord sur New Caprica, puis sur la Terre. Ces deux analepses montrent bien la répétition de la situation : la manière même dont sont filmés les deux plans souligne ce parallélisme, puisqu'il s'agit du même cadrage et de la même scénographie. Toutefois, les circonstances ne sont pas les mêmes, alors que ces deux événements appartiennent au même continuum temporel. Ainsi, la cosmologie de Battlestar Galactica n'est pas stoïcienne. Elle induit même, par ses changements, une perspective cosmologique bien différente.

Certains courants stoïciens avaient déjà envisagé que les cycles temporels puissent être légèrement différents :

Ils [les Stoïciens] sont d'avis qu'après l'embrasement, toutes choses se reproduisent dans le monde, numériquement identiques, de sorte que même l'individu qualifié particulièrement existe et figure identiquement dans ce nouveau monde comme dans le précédent; c'est ce que dit Chrysippe dans son traité en plusieurs livres Du monde [...]. Ils disent aussi qu'entre individus qualifiés particulièrement postérieurs et antérieurs, les seuls écarts concernent certains de leurs accidents extérieurs, écarts qui, affectant le même Dion persistant et vivant, ne le transforment pas. En effet, il ne devient pas un autre homme si, ayant auparavant des grains de beauté sur le visage, plus tard il ne les a plus. Tels sont les écarts, disent-ils, que présentent dans un monde les individus qualifiés particulièrement, par rapport à ceux d'un autre monde ${ }^{12}$.

Les commentateurs Long et Sedley insistent sur la différence majeure entre la conception d'un monde se répétant de manière rigoureusement semblable et celle d'un monde où des différences, même en apparence simplement cosmétiques et mineures, existeraient :

De telles différences, si elles n'affectent en rien l'identité d'un individu, impliquent une cosmologie radicalement différente de celle qu'implique (a). Entre deux mondes, une seule différence de ce type est nécessaire pour qu'ils soient distincts l'un de l'autre. Mais l'occurrence d'une différence quelconque entre deux mondes successifs implique également une différence dans leurs causes antécédentes, et par suite l'abandon de la causalité totalement fermée requise par (a). Peut-être certains Stoïciens ont-ils supposé que l'embrasement nettoyait tous les vestiges de ses antécédents, et laissait à Dieu une matière indéterminée, qui lui permettrait de lancer une séquence causale entièrement nouvelle et indépendante. Sa parfaite rationalité donnerait alors naissance à un monde essentiellement identique, contenant des personnes individuelles numériquement identiques. L'excellence du prochain monde ne serait nullement affectée par la présence ou l'absence de caractéristiques moralement indifférentes, tels que des grains de beauté sur la figure. Et pourtant, ce sont des différences de ce genre qui permettent au prochain monde de se situer dans une dimension temporelle authentiquement différente, puisque Socrate avec un grain de beauté ne pourrait exister en même temps que Socrate sans ce caractère ${ }^{13}$. 
Or, les variations induites par Battlestar Galactica vont bien au-delà. Comme on l'a vu, Leoben explique que les rôles joués tant par les cylons que par les humains peuvent évoluer au sein de ces répétitions. Mais la variation majeure permettant de radicalement transformer la conception de la cosmologie de la série est celle des lieux. Le dernier épisode énumère ces lieux où les répétitions se sont produites : Kobol, la Terre, Caprica. Ainsi, la répétition des événements de Battlestar Galactica répète un schéma fondamental, que l'on peut assimiler au mythe de Frankenstein ${ }^{14}$ - ou celui de la révolte de la créature contre son créateur et de la guerre qui s'ensuit-, mais le récit fait varier les rôles endossés par les personnages dans chaque nouvelle configuration spatio-temporelle.

Ainsi, cette conception de l'éternel retour n'est pas cosmique mais plutôt historique. Les événements narrés par la série ne se déroulent pas dans un monde qui a été autrefois détruit par ekpyrosis et qui est rené de ses cendres identique à lui-même (avec ou sans variantes) mais bien dans un même temps cosmique qui voit se dérouler des cycles historiques à la fois semblables et différents. Cette conception de l'éternel retour n'est donc plus l'expression d'un déterminisme logique et physique, tel que pouvaient l'exposer les stoïciens :

Par «destin», j'entends ce que les Grecs appellent heimarmenè : une ordonnance et une série de causes, puisque c'est la connexion de cause à cause qui d'elle-même produit toute chose ; c'est la vérité perpétuelle, qui s'écoule de toute éternité. Et puisqu'il en est ainsi, rien n'est arrivé qui n'ait dû arriver, de même que rien n'arrivera dont la nature [universelle] ne contienne les causes qui œuvres précisément à sa réalisation. On comprend par là que le destin dont il s'agit n'est pas celui de la superstition, mais celui de la physique, cause éternelle des choses, pour laquelle les choses passées sont arrivées, les choses présentes arrivent, les choses futures arriveront ${ }^{15}$.

17 Il s'agit bien plutôt d'un déterminisme métaphysique et historique. Cette différence permet de réintroduire la possibilité d'un changement, d'une éventuelle liberté mais également de l'échec, c'est-à-dire d'une révolution, au sens étymologique et politique du terme. Elle donne également espoir aux «Cinq Derniers» cylons («Final Five») de pouvoir lutter contre le destin et la fatalité de l'histoire et ainsi « rompre le cycle », ce qui est une thématique essentielle de la fin de la série.

18 Plutôt qu'un temps cosmique circulaire, il nous faut donc plutôt évoquer la possibilité d'un temps cosmique spiralaire, comme dans nombre d'œuvres de science-fiction contemporaine ${ }^{16}$, parmi lesquelles «Les Guerriers du silence » de Pierre Bordage ou «Les Cantos d'Hypérion » de Dan Simmons. En effet, comme dans une spirale, l'histoire est à la fois circulaire et linéaire. Circulaire car elle se répète, mais linéaire en ce que ces répétitions s'effectuent sur fond de variation et tendent à évoluer pour s'approcher d'un centre qui annihilerait la répétition. C'est bien ce que recherchent les Cinq Derniers cylons qui ont tenté et tentent encore de prévenir les humains pour d'éviter la catastrophe. Certes, leur tentative avorte; mais la fin de la série, qui représente le futur de l'époque de Battlestar - c'est-à-dire notre monde contemporain - tend à affirmer que cette fois, le cycle est peut-être brisé et que l'histoire ne se répétera peut-être pas.

Une autre conséquence de cette représentation d'un temps non pas strictement cyclique mais spiralaire est l'importance que revêt la notion de mémoire. En effet, dans la cosmologie stoïcienne, l'on ne se souvient pas des cycles précédents - on les déduit des postulats physiques et logiques de la cosmologie. Ainsi, tout recommence de la même manière sous l'impulsion du principe divin du cosmos, mais rien ni personne, si ce n'est 
les dieux dans certaines interprétations, ne survit à l'ekpyrosis ni ne peut témoigner des cycles précédents. Dans Battlestar Galactica, la nature semi-linéaire du temps spiralaire permet une transmission de la mémoire, d'une part par les textes sacrés de la Pythie, qui accompagnent la présidente jusqu'à la découverte de la Terre, et d'autre part par la mémoire orale des Cinq. Ce rôle central de la mémoire est rendu sensible par les images extraordinaires, notamment dans la troisième saison, où les cartes de navigation stellaire sont combinées aux textes sacrés compulsés par Roslin, les astrogateurs et Adama luimême. Malgré leur imprécision, ces textes sont la mémoire collective de l'humanité qui y cherche les réponses fondamentales à sa survie. La mémoire est également mise en valeur lors des anamnèses des Cinq sur Terre et peu après, lors des quelques séquences où Sam tente de se souvenir de leur passé car il espère y trouver la clé permettant d'expliquer tous les mystères qui les entourent. Et c'est finalement Ellen qui, par sa mémoire individuelle, résout les mystères du passé et donne à ses « enfants » des clés pour le futur. L'importance de la mémoire et du souvenir est centrale dans la série : le temps n'est pas strictement circulaire car, précisément, les Cinq cylons ont conscience de cette répétition, et cette conscience est libératrice. Elle permet d'envisager de sortir du cercle, de «rompre le cycle». On peut ainsi lire le récit comme une réflexion sur le rôle libérateur de la mémoire - mais est-ce à dire que les personnages de Battlestar Galactica sont finalement libres?

La réponse semble négative, et ce, pour deux raisons. D'une part, ces personnages sont bien soumis à un destin qui, contrairement à la cosmologie stoïcienne, se manifeste de diverses manières : par des visions, des rêves prémonitoires partagés, des airs musicaux, des coïncidences troublantes, voire même des anges qui viennent leur parler. Ainsi, le destin semble bien agencé par une puissance transcendante et divine qui manipule les êtres et les événements. La liberté des personnages semble résider à la fois dans leur choix de tenter de déchiffrer ces signes et celui de s'y soumettre. C'est la leçon que ne cesse de donner l'apparition de Numéro Six à Baltar : s'il n'a pas foi en Dieu et s'il ne se soumet pas à son destin, il est perdu. Il s'agit d'un quasi-chantage pour qu'il s'en remette à la providence divine. Cette acceptation peut prendre une forme plus dynamique: certains personnages tentent de retrouver la mémoire et de déchiffrer les signes divins. Kara est véritablement obsédée par l'air que lui faisait jouer son père et par le dessin d'Héra, mais elle exerce sa liberté en cherchant la corrélation possible entre cet air qui la hante, son passé avec son père et le dessin de la petite fille. Et, finalement, elle a l'intuition finale qu'il s'agit des coordonnées de la Terre promise. Ainsi, elle accomplit son destin et exerce la part de liberté qui lui est dévolue et qui est celle du Sage stoïcien, à savoir accepter son destin et l'ordre du monde. Kara choisit d'être le Héraut de la Mort et de guider les hommes vers la Terre promise, de même que Baltar accepte explicitement son destin (S04E05). En effet, ses mots sont très proches de ceux de Sénèque lorsque ce dernier évoque la providence :

Les destins sont nos maîtres et la durée de notre vie est arrêtée dès la première heure de notre naissance. Les causes engendrent leurs effets ; un perpétuel enchaînement détermine les événements particuliers et généraux. S'il faut tout accepter vaillamment, c'est que les choses ne sont pas, comme nous nous le figurons, des hasards, mais des résultats. Il y a longtemps que toutes nos joies et toutes nos larmes sont décrétées, et, si grande que puisse être la diversité apparente des existences individuelles, tout se ramène à un principe unique : éphémères, nous sommes voués à l'éphémère. Alors à quoi bon s'insurger? Pourquoi se plaindre? C'est la loi même de la vie. Que la 
nature fasse ce qu'elle veut des corps qui lui appartiennent; nous, allègres et courageux quoi qu'il arrive, ne perdons pas de vue que de ce qui périt rien n'est nous-mêmes. Que fait l'homme de bien ? Il se livre au destin ${ }^{17}$. ils sont déterminés par leur être moral et ce que l'on nommerait aujourd'hui leur psychologie. En effet, les stoïciens avaient réintroduit une part de responsabilité morale en distinguant l'impulsion du destin et notre faculté de délibération :

\begin{abstract}
«De même, dit-il, que si tu pousses un cylindre de pierre sur un plan fortement incliné, tu as produit la cause et le commencement de son mouvement vers l'avant, mais que bientôt il roule vers l'avant, non plus du fait de ton impulsion, mais du fait que sa forme et sa disposition à rouler sont telles, de même l'ordre, la raison d'être et la nécessité du destin mettent en mouvement les types réels de causes et leurs commencements, mais l'impulsion délibérative de notre esprit et nos actions effectives sont contrôlées par notre volonté et notre intellect propres. » En accord avec cela, il dit, et ce sont ses propres termes : "C'est pourquoi les Pythagoriciens ont raison de dire "apprends que les hommes ont choisi leurs propres troubles ", entendant par là que le dommage qu'ils subissent est entre les mains de chacun, et que c'est en suivant leur impulsion, leur propre état d'esprit et leur propre disposition qu'ils font fausse route et subissent du dommage. » Pour cette raison, il refuse que ceux qui, du fait de leur paresse ou de leur méchanceté, sont nuisibles et irréfléchis, soient tolérés et entendus si, quand on les prend sur le fait, ils se réfugient dans la nécessité du destin, comme si c'était l'asile d'un temple, quand ils disent que les pires de leurs méfaits sont attribuables au destin, et non à leur propre méchanceté ${ }^{18}$.
\end{abstract}

Mais cette faculté de délibération est entièrement conditionnée par l'être moral de chacune ou chacun. Les héros de Battlestar Galactica peuvent parfois avoir une illusion de liberté, à la manière de la pierre de Spinoza ${ }^{19}$, mais ils sont guidés par un déterminisme intérieur qui les dépasse et qui est représenté grâce à un effet de lecture jouant sur l'intertextualité.

23 L'intertextualité mythico-épique à l'œuvre dans la série enferme les personnages dans une détermination qui naît d'un effet de lecture. En effet, leurs liens avec l'hypotexte mythique et épique les surdétermine et les constitue en archétypes qui tendent à agir toujours de la même manière face à une situation donnée. Ainsi, le déterminisme du caractère moral stoïcien est mimé par un effet de lecture intertextuelle qui donne naissance à des effets de répétition et d'échos culturels produisant chez les spectateurs une impression de « déjà-vu » propre à l'éternel retour. Par exemple, malgré ses grandes déclarations lyriques, Baltar agit toujours par opportunisme et pour son autopréservation; le colonel Tigh, même lorsqu'il a découvert sa nature de cylon, continue à aboyer et à menacer de passer les cylons par le sas (etc.). Cette récurrence des situations tend à nous faire sentir que les personnages sont bien déterminés par leur être intérieur et agissent nécessairement, de manière "programmée ", répondant à leur destinée et à la providence divine ${ }^{20}$.

Comme le genre de la série télévisée repose sur un principe programmé de récurrence, à l'œuvre dans le déroulement de l'intrigue, le développement des personnages, la mise en image ou en son $^{21}$, ce principe de récurrence réflexif renforce l'impression que les événements se répètent, mimant ainsi, à échelle microscopique, le cycle de l'éternel 
retour. De surcroît, la construction mythique de la série contribue à des effets de lecture intertextuels : en effet, Battlestar Galactica se présente comme le récit d'un mythe fondateur, celui de notre monde. La série correspond donc très précisément à l'une des définitions du mythe que donne Mircéa Éliade :

Le mythe raconte une histoire sacrée; il relate un événement qui a eu lieu dans le temps primordial, le temps fabuleux des «commencements». Autrement dit, le mythe raconte comment, grâce aux exploits des Êtres Surnaturels, une réalité est venue à l'existence, que ce soit la réalité totale, le Cosmos ou seulement un fragment: une île, une espèce végétale, un comportement humain, une institution. C'est donc toujours le récit d'une création $^{22}$. intertexte antique de la série. L'onomastique, d'abord, crée un effet de « déjà-vu » propre à mimer l'éternel retour: les noms des douze grands dieux de Kobol, ainsi que les allusions à la Pythie et les surnoms mythologiques comme Apollon, Héra ou Athéna contribuent à cet effet. De plus, les noms sont également une manière d'enfermer les personnages dans une détermination liée à leur sens ou leur consonance mythique. Ainsi, le lieutenant (puis capitaine) Agathon est, par essence, un homme bon qui ne sait pas prendre de décision moralement mauvaise. C'est lui qui, le premier, va percevoir l'humanité des cylons et l'accepter, c'est lui qui va refuser de fermer les yeux sur la xénophobie envers les Sagittarons et c'est également lui qui va saboter le plan d'extermination génocidaire des cylons pourtant adopté par la flotte, la présidente et l'Amiral Adama d'un commun accord. Ellen Tigh peut être lue comme l'incarnation d'Hélène de Troie, une mangeuse d'hommes toujours prompte à semer la discorde. L'onomastique est simultanément de nature biblique : l'Amiral Adama est ainsi le père spirituel de la flotte, c'est-à-dire de ce qui survit de l'humanité; Saul Tigh, homme belliqueux et compagnon de Samuel Anders qui deviendra finalement l'Hybride (figure Cylon prophétique qui pilote le vaisseau) fait écho au Saül de l'Ancien Testament, grand roi-guerrier qui succéda au prophète Samuel.

Outre l'onomastique, l'intrigue générale de Battlestar Galactica est une réécriture tenant à la fois de l'Iliade et de l'Énéide. Iliade car la flotte est en quelque sorte «assiégée » par les cylons pendant une majeure partie de la série. La répétition des sorties des «vipers » et les combats épiques ne sont pas si différents des sorties homériques et leur succession de combats, de morts et de funérailles. Mais la série fonctionne également, et peut-être surtout, comme une réécriture de l'Énéide en tant qu'elle est l'histoire des réfugiés d'une civilisation disparue. C'est ainsi que l'on peut lire l'arrêt sur New Caprica comme un clin d'œil à l'épisode carthaginois de l'épopée virgilienne. Lee Adama, le capitaine honorable, fils de l'Amiral - qui peut être également vu comme un nouveau Roi Priam - respectant 
ses parents, l'idée de démocratie et de justice et les dieux, peut être considéré comme un nouveau pius Aeneas. Plus discrètement, il est fait mention du passé de Boomer dans la première saison : cette dernière viendrait d'une colonie nommée Troie. Ne peut-on, dès lors, assimiler Boomer au cheval de Troie ? Car Boomer est bien celle qui s'infiltre chez l'ennemi afin de le détruire de l'intérieur, et ce à plusieurs reprises. Enfin, le personnage de Kara Thrace fonctionne plus implicitement comme le plus célèbre des personnages homériques : son surnom, «Starbuck » la désigne comme une femme virile et efface son caractère féminin ${ }^{23}$. Elle est blonde ; c'est le meilleur pilote de la flotte; elle se définit par son caractère colérique; elle est toujours prête à en découdre, dans l'espace comme sur terre; enfin, elle n'a pas peur de la mort, mais craint d'être oubliée. Tout la désigne comme l'avatar d'Achille, dans les moindres détails: elle est versée dans les arts puisqu'elle a appris le piano et peint; elle a été élevée par sa mère qui lui répète qu'elle a un destin particulier; elle est blessée (au genou plutôt qu'au talon); elle est désignée comme le Héraut de la mort, ce qui peut la rapprocher de l'épithète homérique « tueuse d'hommes".

Ainsi, l'intertextualité redouble le déterminisme moral qui guide les personnages : ceux-ci sont déterminés par leur modèle mythico-épique. Ils incarnent, pendant toute la série, ce modèle ; ainsi, leurs décisions sont chaque fois motivées par leur être moral, inscrit dans leur lien intertextuel avec ces grands personnages épiques ou mythiques, ces marqueurs intertextuels renforçant l'effet de « déjà-vu » mimant l'éternel retour et la circularité du temps.

Pour finir, ce temps cyclique, ou plutôt spiralaire, fonctionne comme un avertissement : l'histoire pourrait à nouveau se répéter. Il s'agit là d'une mise en garde classique dans la science-fiction contre le développement d'une technologie échappant à son créateur. Ici, la dimension cyclique de la série rend cet effet de mise en garde particulièrement efficace. Ainsi, certains dialogues résonnent avec une inquiétante étrangeté à nos oreilles. On peut penser notamment au discours quasi transhumaniste de Cavill sur la finitude et l'imperfection de l'homme (S04E15) ou à celui de Numéro Six devant le Quorum, affirmant que l'humanité ne doit sa complétude qu'à sa finitude et sa nature mortelle (S04E07). Plus subtilement que par l'avertissement final, ces scènes réactivent les débats actuels sur la nature de l'être humain, le rôle de la technologie dans l'évolution humaine, ou la naissance imminente d'une posthumanité. Dans le dernier épisode, lorsque Numéro Six exprime l'espoir que cette fois le cycle de la violence génocidaire puisse être rompu, elle nous invite à lire, dans une métalepse, la série Battlestar Galactica comme une vision prémonitoire prévenant (à travers les spectateurs) toute l'humanité du danger qui la guette, et mobilisant pour cela l'ensemble des croyances humaines passées et présentes, des oracles grecs aux mandalas bouddhistes, aux prophètes ou anges chrétiens.

\section{BIBLIOGRAPHIE}

BATAILLE Sylvaine, «Les pièges du temps : la réappropriation de l'Antiquité gréco-latine dans Battlestar Galactica », GRAAT online $n^{\circ} 6$, Les Pièges des nouvelles séries télévisées américaines, éd. Sarah 
Hatchuel et Monica Michlin, déc. 2009, http://www.graat.fr/backissuepiegesseriestv.htm (consulté le 07 juillet 2017).

ELIADE Mircea, Aspects du mythe, Paris, Gallimard, coll. Folio, 1963.

GOURINAT Jean-Baptiste, «Éternel retour et temps périodique dans la philosophie stoïcienne », Revue philosophique de la France et de l'étranger 2002/2 (Tome 127), p. 213-227, https:// www.cairn.info/revue-philosophique-2002-2-page-213.htm (consulté le 07 juillet 2017).

HATCHUEL Sarah, Rêves et séries américaines, la fabrique d'autres mondes, Aix-en-Provence, Rouge Profond, 2016.

LONG Anthony Arthur et David SEDLEY, Les philosophes hellénistiques Tome II : Les Stoïciens, tr. Jacques Brunschwig et Pierre Pellegrin, Paris, Garnier-Flammarion, (1987), 2001.

SÉNÈQUE, De providentia, tr. René Waltz, Paris, Les Belles Lettres, coll. Classiques en poche, (2002), 2011.

\section{NOTES}

1. En effet, les coïncidences et certaines destinées comme celle de Kara semblent affirmer l'existence d'un « grand architecte » (ou d'un Auteur) de l'ensemble de la diégèse.

2. Sur l'héritage gréco-latin dans Battlestar Galactica, voir Sylvaine Bataille, « Les pièges du temps : la réappropriation de l'Antiquité gréco-latine dans Battlestar Galactica », GRAAT online n6, éd. Sarah Hatchuel et Monica Michlin, 2009. [URL]: http://www.graat.fr/ backissuepiegesseriestv.htm (consulté le 07 juillet 2017).

3. Jean-Baptiste Gourinat, "Éternel retour et temps périodique dans la philosophie stoïcienne ", Revue philosophique de la France et de l'étranger 2002/2 (Tome 127), p. 213-227, https:// www.cairn.info/revue-philosophique-2002-2-page-213.htm (consulté le 07 juillet 2017).

4. Le tagline de la série à propos des cylons est, rappelons-le : « There are twelve models and they have a plan. »

5. La quête de la Terre sous-tendant tout le récit, toute « révélation » se doit d'être partielle pour ne pas trop « dévoiler » la fin.

6. Voir le chapitre de Sarah Hatchuel sur Battlestar Galactica in Rêves et séries américaines, la fabrique d'autres mondes, Paris, Rouge Profond, 2016.

7. Némésius, 309, 5 - 311, 2 in Anthony A. Long et David Sedley, Les philosophes hellénistiques Tome II : Les Stoïciens, tr. Jacques Brunschwig et Pierre Pellegrin, Paris, Garnier-Flammarion, (1987) 2001, p. 325.

8. Cicéron, Du destin 28 - 30, in Long et Sedley, op. cit., p. 390.

9. Cicéron, Du destin 39 - 43, in Long et Sedley, op. cit., p. 475.

10. On peut penser à l'Hymne à Zeus de Cléanthe.

11. Ce qui pose la question du rôle de Numéro Six: manipule-t-elle Baltar et le cours des événements ou est-elle réellement une " prophétesse »?

12. Alexandre d'Aphrodise, Sur les Analytiques premiers d'Aristote, 180, 33 - 36 et 181, $25-31$, in Long et Sedley, op. cit., p. 328.

13. Long et Sedley, op. cit., p. 333.

14. Remarquons l'allusion à Mary Shelley dans le nom de la clone de Numéro Six «Shelley Godfrey ».

15. Cicéron, De la divination I, 125 - 126, in Long et Sedley, op. cit., p. 385.

16. On peut se demander si l'idée même d'un temps cosmique circulaire et déterministe nous est devenu si étranger et si insupportable que les auteurs de S-F doivent l'adapter et le transformer 
en un temps spiralaire qui admet à la fois la linéarité du changement, du progrès et de la liberté et la circularité fatale que les héros doivent chercher à briser.

17. Sénèque, De providentia, V, 7 et 8,tr. René Waltz, Paris, Les Belles Lettres, coll. Classiques en poche, (2011) 2002, p. 109.

18. Aulu-Gelle (citant Chrysippe), VII, 2, 6-13 in Long et Sedley, op. cit., p. 479.

19. Spinoza, Lettre LVIII dite Lettre à Schuller.

20. On pourrait aussi dire, bien sûr, dans une perspective "posthumaine ", qu'ils "sont programmés pour cela ».

21. Sur ces questions de récurrence, voir Isabelle Périer, "The Tudors ou quand l'histoire se répète", Colloque Sens et motifs de la récurrence dans les séries télévisées, 15 septembre 2011, Université Paris-Sorbonne. (à paraître).

22. Mircea Eliade, Aspects du mythe, Paris, Gallimard, coll. Folio, 1963, p. 16.

23. On se rappellera également que Starbuck était un homme dans la version première de la série par Glen Larson (ABC, 1978-1979).

\section{RÉSUMÉS}

Cet article se propose d'étudier la conception du temps, du destin et de la liberté à l'œuvre dans le reboot par Ronald D. Moore de la série télévisée Battlestar Galactica (Syfy, 2003-2009) en la confrontant à la cosmologie cyclique et providentielle des stoïciens. Dans la mesure où l'intertextualité à l'œuvre dans la série est une manière de représenter les déterminismes internes et externes pesant sur les personnages et de redoubler, par un effet de "déjà-vu ", l'impression d'un éternel retour, nous nous appuierons aussi sur une analyse mythocritique pour saisir les enjeux philosophiques et cosmologiques de ce récit, entre destin et liberté.

This article explores how time, destiny and freedom are represented in Ronald D. Moore's reboot of Battlestar Galactica (Syfy, 2003-2009) by comparing it with the cyclical and providential cosmology of Stoic philosophers. Since the series' intertextuality increases the effect of "déjà $v u$ ", to give the impression of the eternal return of the same events, we will read the paradoxes of the series through a mythocritical frame of analysis as well as a philosophical one.

\section{INDEX}

Keywords : Battlestar Galactica, destiny, cyclical time, freedom, religion, science fiction, spirituality, stoicism

Mots-clés : Battlestar Galactica, destin, liberté, religion, science-fiction, spiritualité, stoïcisme, temps cyclique

\section{AUTEUR}

\section{ISABELLE PÉRIER}

Agrégée de Lettres classiques et docteure en Littérature comparée, Isabelle Périer enseigne dans un lycée à Argenteuil. Ses domaines de recherche ont principalement trait à la culture populaire 
et notamment à la science-fiction, la fantasy, la littérature de jeunesse, les narrations sérielles et le jeu de rôle sur table.

Isabelle Périer holds an Agrégation in Classics and a PhD in Comparative Literature and teaches in a high school in Argenteuil. Her research focuses on popular culture, particularly on sciencefiction, fantasy, young adult literature, serial narratives and tabletop RPG. 